\title{
The uncertain science of predicting tuberculosis
}

${ }^{1}$ Toyin Togun, MD, MPH, PhD

${ }^{1,2}$ Madhukar Pai, MD, PhD

${ }^{1}$ McGill International TB Centre and Department of Epidemiology and Biostatistics, McGill University, Montreal, Canada

${ }^{2}$ Manipal McGill Center for Infectious Diseases, Manipal University, Manipal, India

Prof Madhukar Pai, MD, PhD

McGill University

Dept of Epidemiology \& Biostatistics

1020 Pine Ave West

Montreal, QC H3A 1A2, Canada

Tel: 514-398-5422

Fax: 514-398-4503

Email: madhukar.pai@mcgill.ca 
An estimated quarter of the world's population is infected with Mycobacterium tuberculosis. ${ }^{1}$ Unfortunately, currently available immunodiagnostic tools are unable to discriminate the stages within the spectrum of tuberculosis infection. ${ }^{2,3}$ Neither the tuberculin skin test (TST) nor interferon-gamma release assays (IGRAs) meets the need for a highly predictive test that can identify the latently infected persons who are at increased risk for the development of active TB disease and would therefore benefit most from preventive therapy. ${ }^{4}$ This limitation, as well as the high prevalence of latent TB infection (LTBI), makes it daunting for high burden countries to address latent TB in their control programs. ${ }^{5}$

While the search for new predictive biomarkers and biosignatures is starting to show promise ${ }^{6}$, are there ways to squeeze more predictive value out of existing tests? One way to maximize the predictive value of existing LTBI test is to make sure that persons at low risk for TB infection are not screened. While this recommendation is already a part of guidelines, ${ }^{7}$ many low-risk persons still get screened in practice. A good example is the widespread screening of low-risk healthcare workers in North America, a practice that is already posing challenges. ${ }^{8}$

Another approach to enhance predictive utility is to use multivariable risk prediction models that incorporate clinical and epidemiological risk factors (e.g. age, history of contact, HIV infection, immunosuppressive medications) with test results. ${ }^{9}$ Online risk calculators (The Online TST/IGRA Interpreter; http://www.tstin3d.com/) have made this feasible.

A third approach is to use serial (repeated) rather than cross-sectional testing. ${ }^{9}$ It is well known that persons who recently converted their TST are at high risk of progression. Using the same logic, individuals who convert their IGRA should be at a higher risk of developing TB disease. Although limited, there is some evidence to support this hypothesis. ${ }^{10}$

In this issues of the Lancet Respiratory Medicine, Andrews and colleagues analysed longitudinal data from a cohort of 2,512 BCG-vaccinated, Quantiferon-TB Gold In-Tube (QFT)-negative and HIV-uninfected South African infants recruited into a TB vaccine trial. ${ }^{11}$ They investigated the association between IGRA conversions (i.e. increase in IFN- $\gamma$ values determined by serial QFT testing) and the risk of subsequent development of active TB disease. The results showed that QFT converters at IFN- $\gamma$ values $>4.00 \mathrm{IU} / \mathrm{ml}$ had a significantly higher disease incidence compared to both non-converters (IRR 42.5; $p<0.0001$ ), and to converters at IFN- $\gamma$ values between $0.35-4.00 \mathrm{IU} / \mathrm{ml}$ (IRR 11.4; $p<0.00047)$.

So, the results suggest that a big spike in IFN- $\gamma$ levels is strongly associated with risk of TB. But does the spike predict 'future' risk of TB disease, or is it a consequence of subclinical or incipient disease? While $10 / 63$ children with QFT conversion (threshold of IFN $-\gamma>4.00 \mathrm{IU} / \mathrm{ml}$ ) were diagnosed with TB disease (28.0 cases per 100 person-years), the median time to diagnosis among these QFT converters from the time of QFT testing was merely 44 days. Thus, one can make a case that the IFN- $\gamma$ levels spiked as these children were developing active TB. Therefore, an increase in IFN- $\gamma$ levels is not really predictive of future risk progression to active TB but it is the outcome of imminent development of sub-clinical or incipient TB disease.

Another recent study in the same South African setting suggested that a 16-transcript whole-blood RNA signature might prospectively identify adolescents at risk of developing active TB disease. ${ }^{6}$ The predictive potential of the TB risk signature was highest in the 6-month period immediately before diagnosis, and less predictive over longer follow-up periods. Taken together, both biomarker studies appear to be identifying those with subclinical or incipient disease over the short-term, rather than truly 
predict future TB development. We agree with Cobelens and colleagues who call these 'incipient tuberculosis tests', as compared to 'persistent infection tests'. ${ }^{12}$

This distinction is critical because it is hard to see how preventive therapy can be given to children who may already have subclinical disease, or on the verge of developing active TB within a short period of time. Needless to say, preventive therapy might be inadequate and possibly dangerous in this context. As such, instead of isoniazid preventive therapy, healthcare workers could use a spike in IFN-g levels to aggressively investigate and treat for active TB disease. However, the cost-effectiveness and real world applicability of serial IGRA testing, or blood gene signature assays, in high burden countries is doubtful. Considerable translational work is required to develop simpler, affordable, predictive assays that can be deployed by TB control programs in low resource settings, and target product profiles for such tests have been drafted. ${ }^{13}$

While the science of TB prediction remains uncertain, there is no uncertainty about the high burden of TB in South Africa, even in young children. In this study, about 7\% of infants had QFT conversions after a year, suggesting a very high annual risk of TB infection in this community. This, in turn, suggests poor control of TB among adults, and ongoing transmission in the community. Clearly, much more needs to be done to protect children from TB in South Africa and globally.

Conflicts of interest: The author declared no conflicts of interest

\section{Bibliography}

1. Houben RM, Dodd PJ. The Global Burden of Latent Tuberculosis Infection: A Re-estimation Using Mathematical Modelling. PLoS Med 2016; 13(10): e1002152.

2. Pai M, Behr M, Dowdy D, et al. Tuberculosis. Nature Rev Dis Primers 2016; 2: 1-23.

3. Barry CE, 3rd, Boshoff HI, Dartois V, et al. The spectrum of latent tuberculosis: rethinking the biology and intervention strategies. Nat Rev Microbiol 2009; 7(12): 845-55.

4. Rangaka MX, Wilkinson KA, Glynn JR, et al. Predictive value of interferon-gamma release assays for incident active tuberculosis: a systematic review and meta-analysis. Lancet Infect Dis 2012; 12(1): 4555.

5. Pai M, Sotgiu G. Diagnostics for latent tuberculosis infection: incremental, not transformative progress. Eur Resp Journal 2016; 47(3): 704-6.

6. Zak DE, Penn-Nicholson A, Scriba TJ, et al. A blood RNA signature for tuberculosis disease risk: a prospective cohort study. Lancet 2016.

7. Lewinsohn DM, Leonard MK, LoBue PA, et al. Official American Thoracic Society/Infectious Diseases Society of America/Centers for Disease Control and Prevention Clinical Practice Guidelines: Diagnosis of Tuberculosis in Adults and Children. Clin Infect Dis 2017; 64(2): 111-5.

8. Moses MW, Zwerling A, Cattamanchi A, et al. Serial testing for latent tuberculosis using QuantiFERON-TB Gold In-Tube: A Markov model. Sci Rep 2016; 6: 30781.

9. Pai M, Denkinger CM, Kik SV, et al. Gamma Interferon Release Assays for Detection of Mycobacterium tuberculosis Infection. Clinical microbiology reviews 2014; 27(1): 3-20.

10. Machingaidze $\mathrm{S}$, Verver $\mathrm{S}$, Mulenga $\mathrm{H}$, et al. Predictive Value of Recent QuantifERON Conversion for Tuberculosis Disease in Adolescents. Am J Respir Crit Care Med 2012; 186(10): 1051-6. 
11. Andrews JR, Nemes E, Tameris M, et al. Serial QuantiFERON testing and tuberculosis disease risk among young children: an observational cohort study Lancet Respir Med 2017.

12. Cobelens F, Kik S, Esmail H, Cirillo DM, Lienhardt C, Matteelli A. From latent to patent: rethinking prediction of tuberculosis. Lancet Respir Med 2016.

13. NDWG LTBI Task Force of the Stop TB Partnership's New Diagnostics Working Group. Draft Target Product Profile (TPP) for a test for progression of tuberculosis infection. URL:

http://www.finddx.org/wp-content/uploads/2016/05/TPP-LTBIprogression.pdf (accessed 19 Jan 2017). 2016. 